\title{
Incidentally detected isolated Aspergillus fumigates infection of the renal allograft
}

\author{
Kajal N. Patel ${ }^{1 * \oplus}$, Nitin A. Pate ${ }^{2 \oplus}$, Shruti P. Gandhi ${ }^{\oplus}{ }^{\oplus}$, Hargovind L. Trivedi ${ }^{3}$
}

\begin{abstract}
Fungal infection represents $5 \%$ of the infections of post renal transplant recipients. The frequency of invasive Aspergillus ranges from $0.5 \%$ to $2.2 \%$ with a mortality rate of $88 \%$. In renal transplantation, Aspergillus infection usually affects primarily the lungs with occasional dissemination and the central nervous system. Involvement of a renal allograft in the isolated form is rare. A-35-year-old male post-renal transplant patient presented in our institute for routine follow up examination. Ultrasound and computed tomography (CT) were conducted in our radiology department, suggestive of abscess formation in mid pole of transplanted kidney. The patient did not have any clinical symptoms. His serum creatinine level was also within normal limit. Diagnosis of Aspergillus fumigates was made by aspiration of pus. Treatment started according to culture and sensitivity report. Ultimately graft nephrectomy was performed to control infection. Aspergillus infection of a renal allograft remains a key issue for nephrologists and infection specialists. For diagnosis of fungal infection, a high index of suspicious is necessary. In the present case, the infected allograft nephrectomy and the elimination of immune-compromised state and the prompt administration of antifungal therapy, made recovery possible. However, early diagnosis remains difficult.

Keywords: Aspergillus fumigatus, Fungal infection, Kidney transplant, Aspergillosis

Citation: Patel KN, Patel NA, Gandhi SP, Trivedi HL. Incidentally detected isolated Aspergillus fumigates infection of the renal allograft. J Renal Endocrinol. 2021;7:e08. doi: 10.34172/jre.2021.08.

Copyright $\odot 2021$ The Author(s); Published by Nickan Research Institute. This is an open-access article distributed under the terms of the Creative Commons Attribution License (http://creativecommons.org/licenses/by/4.0), which permits unrestricted use, distribution, and reproduction in any medium, provided the original work is properly cited.
\end{abstract}

\section{Introduction}

Infections are the second highest cause of mortality in kidney transplant (KT) recipients. The incidence of fungal infections depends on the type of organ transplantation, $5 \%$ to $17 \%$ in heart transplantation, $14 \%$ to $22 \%$ in simultaneous heart-lung transplant, $2 \%$ to $42 \%$ in liver transplantation, $2 \%$ to $14 \%$ of kidney transplantation (1). Species of the Aspergillus family account for the majority of these infections and Aspergillus fumigatus in particular, may be regarded as the most important air borne saprophytic fungus $(2,3)$. Fungal infection has a tendency for invading blood vessels, both small and large, both arteries and veins, causes thrombosis and infarction. Aspergillus infection incidence is $0.5 \%$ to $2.2 \%$ in post renal transplant recipients and mortality is nearly $88 \%$ $(4,5)$. The majority of post-transplant recipients with Aspergillus infection have pulmonary (6), rhino-cerebral or disseminated infection. Infection of a single organ without a novert site of origin has been rarely reported. The renal lesions are characterized pathologically by multiple parenchymal micro abscesses, indicative of hematogenous spread to the organ. We report a case of incidentally detected isolated Aspergillus fumigates infection of renal allograft without evidence of site of origin.

\section{Study of a patient}

A-35-year-old non-diabetic male patient was come to our institute for routine follow up of post-renal transplantation after 4 months. The patient had basic end-stage renal disease due to solitary contracted ectopic kidney in right iliac fossa. The patient had history of living related renal transplant in left renal fossa with mother as a donor. Their maintenance immunosuppression regimen was prednisolone, tacrolimus and mycophenolate mofetil. $\mathrm{He}$ was also taking fluconazole, Sulfamethoxazole, bacteriostatic antibiotic and valganciclovir for three months. The post operative course was uncomplicated. His serum creatinine ( $\mathrm{sCr}$ ) level was stable $1.25 \mathrm{mg} / \mathrm{dL}$ at time of discharge.

During routine follow up examination after four months of post renal transplantation, basic investigation was done in our institute. The transplanted graft was non-tender on palpitation. Serum creatinine level was within normal limit. His ultrasonography and color Doppler study of 
Implication for health policy/practice/research/ medical education

Incidence of fungal infections is only $5 \%$ among the total infections of post-renal transplant recipients. In renal transplantation, Aspergillus infection usually affects primarily the lungs with occasional dissemination and the central nervous system. Involvement of a transplanted kidney in the isolated form is rare. The notable aspect of the case is lack of clinical symptoms.

graft vessels were done in our radio diagnosis department. It displayed normal size transplanted kidney in left renal fossa with about $3.1 \times 2.8 \mathrm{~cm}$ size hypoechoic lesion in mid pole without vascularity (Figure 1 ). There were no hydronephrosis and normal resistive index (RI) within graft. The patient did not have history of fever, pain, cough or dyspnea. Provisional diagnosis of non-liquefied abscess was kept. The plain chest film was normal. Blood, urine and sputum cultures yielded negative results. Due to non-liquefied smaller sized lesion, aspiration of lesion could not be possible at time of presentation. Therefore, the patient was treated with antibiotic of cefoperazone and sulbactam. Close follow up of lesion was conducted by ultrasonography and blood investigations. During this period patient also underwent plain CT scan and MRI study of abdomen, findings were consistent with USG (Figure 2). Ultimately aspiration of lesion of transplanted kidney became possible after 3 months of diagnosis of

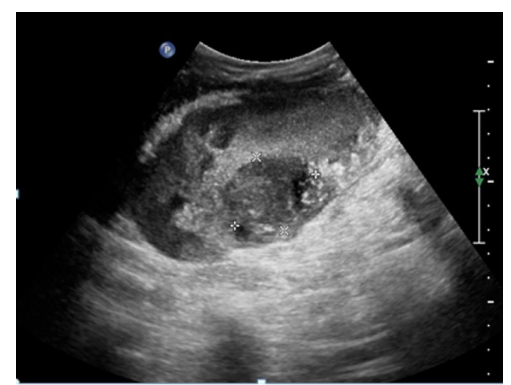

Figure 1. Ultrasonography image of transplanted kidney shows well defined hypoechoic lesion in cortex of mid pole

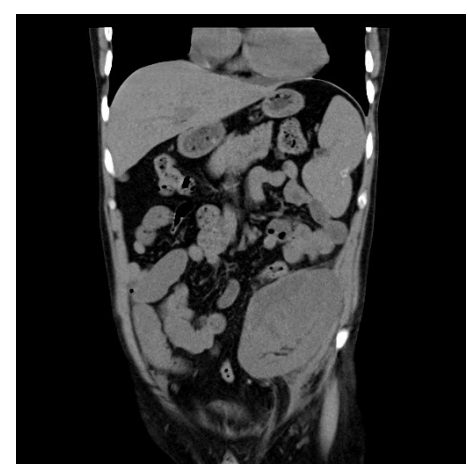

Figure 2. Coronal image of plain CT scan of abdomen shows well defined hypodense subcapsular collection involving mid pole of transplanted kidney in left renal foosa. renal allograft abscess. At that time lesion appeared quite enlarge measuring about $5.2 \times 2.8 \mathrm{~cm}$ with sub-capsular extension at mid pole with elevated mean RI of about 0.81 and $\mathrm{sCr}$ of $2.37 \mathrm{mg} / \mathrm{dL}$. Fluid cytology of aspirated pus suggested heavy growth of Aspergillus fumigates organism. Culture and sensitivity of pus showed very sensitive voriconazole and resistant amphotericin-B drug. Thereby treatment with voriconazole $200 \mathrm{mg}$ two times a day was started but the patient had same size abscess in renal allograft and his sCr level was increased day by day and ultrasonography also did not indicate resolving abscess. Finally, transperitoneal laparoscopic deroofing of subcapsular collection and subsequent allograft nephrectomy was conducted after two months of diagnosis of fungal abscess. Gross specimen of transplanted kidney shows few area of necrosis at cortex (Figure 3). Microscopic findings also suggested Aspergillosis without ureteric extension or immune injury (Figure 4). After graft nephrectomy patient was improved. The patient was clinically stable on hemodialysis without infection within graft bed or any other site during three months follow up.

\section{Discussion}

There has been a dramatic increase in the incidence of aspergillosis in the last two decades, yet the mortality has decreased from 92\% (between 1990 and 1995) to 60\% (between 1998 and 2001) (7). Aspergillus spores are ubiquitous and can be cultured from stored grains, most soil and vegetation, the organisms can be a laboratory

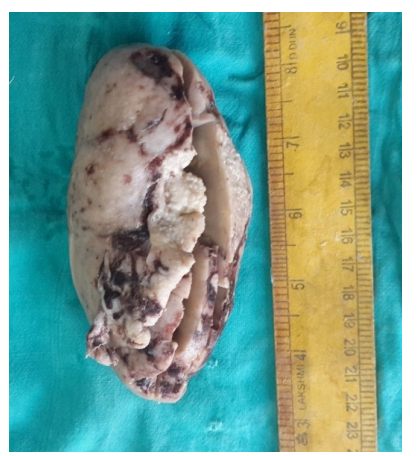

Figure 3. Gross specimen of transplanted kidney show few area of necrosis within cortex.

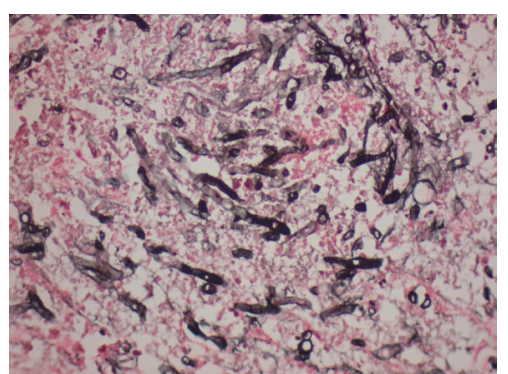

Figure 4. Histopathology showing tubular septate branching hyphae surrounded by polymorphonuclear cells, cell debris and lymphoplasmacytic cells (hematoxylin and eosin, $\times 400$ ) consistent with Aspergillus. 
contaminant, an allergic stimulant or a secondary colonizer if lung cavities (8).

\section{Risk factors}

Fungal infections are more common between the first and sixth month after post-transplantation but they may appear earlier also (9). The use of immunosuppressive agents represents important predisposing factors for fungal infection, especially steroids. Other commonly related factors include diabetes mellitus, renal failure, hematological disease, broad spectrum antibiotics, indwelling catheters, increasing number of surgical procedures, infection with cytomegalovirus, exposure to infective fungi, chronic liver disease, prolong pre and post-transplant dialysis $(10,11)$. As per past record of infections, the presence of Aspergillus species in the environment is the most important factor in nosocomial invasive Aspergillosis.

\section{Diagnosis}

Diagnosis of Aspergillus infection has been based on evidence of fungal invasion in tissue biopsies. The aspergillosis is identified by their uniform, tubular and regularly septate hyphae with progressive and dichotomous branching. Renal transplant ultrasonography may be normal in earlier stage or show non-vascular hypo/iso-echoic lesion, which is a non-specific finding. Imaging studies like contrast enhanced computed tomography(CECT) may help in the diagnosis of this fungal infection within transplanted kidney showing presence of perinephric collection suggesting intra-renal abscess and no or poor excretion of contrast.

Since Aspergillus species are respiratory pathogens, the most common form of infection is pneumonia, followed by sinusitis (12). Weiland et al, in a review of aspergillosis in renal transplant patients, describe three characteristic patterns 1) cavitary lung disease 2) diffuse pulmonary disease, and 3) central nervous disease always after a prior pulmonary infection (8). HRCT thorax scan could be useful for detecting a hypothetic pulmonary infiltrate in the present patient with normal X-ray. As another possibility, the fungus may have been seeded from sinuses. However our patient neither had lung disease or sinusitis. Although fungal endocarditis is uncommon in kidney transplant patients, it was also investigated. Nonetheless, 2-D echocardiography of our patient was normal. Because of the single kidney infection without evidence of a site of origin as in our case, we can speculate on transmission of the fungus together with the allograft. Donor-to-host transmission of infection is well documented in many case reports but they often do not differentiate donor-related transmission from iatrogenic contamination during preservation. In fact, the donor is a less likely source of contamination. Perfusate solution offers an excellent medium for microbial growth. In a recent review by
Gottesdiener et al (13), fungi accounted for 0.2\%-2.5\% of the isolates in cultures taken from perfusion solutions used for kidney preservation. Regarding the source of graft infection in our case, extensive investigation failed to reveal any origin of fungal infection.

After the introduction of voriconazole drug for treatment of fungal infection, it shows that mortality and morbidity is decreased in comparison with amphotericin B. The enhancement of the immune system with immunomodulatory agents like interferon-gammas an attractive alternative or adjuvant to established antifungal therapy (14). With any kind of antifungal treatment used, the cure rate depends on how early treatment is initiated. In the present case, the nephrectomy of infected allograft and thus elimination of immunocompromised state and the prompt administration of antifungal therapy made recovery possible.

\section{Conclusion}

Aspergillus infection of a renal allograft remains a key issue for nephrologists and infection specialists. The presence of an isolated aspergillus abscess within a renal allograft without evidence of systemic infection as in our case is very rare. The notable aspect of our case is lack of clinical symptoms. To improve prognosis, a high index of suspicious is necessary. Accurate information regarding colonization and the antifungal susceptibility of the isolated organisms is required to start suitable empirical treatment. However, early diagnosis remains difficult.

Authors' contribution

KNP and SPG were the principal investigators of the study. KNP and NAP had a major role in preparing the concept and design. KNP, NAP and SPG participated in preparing the final draft of the manuscript and revised the manuscript. All authors have read and approved the content of manuscript and confirmed the accuracy and integrity of any part of the work.

\section{Acknowledgments}

We are also thankful to our librarian Jyotsana Suthar for the literature search and submission.

Conflicts of interest

The authors declared no conflicts of interest with respect to the authorship and/or publication of this article.

\section{Ethical considerations}

Ethical issues (including plagiarism, data fabrication, double publication) have been completely observed by the authors. The patient gave the consent to publish as a case report.

\section{Funding/Support}

None.

\section{References}

1. Patel R, Paya CV. Infections in solid organ transplant recipients. Clin Microbiol Rev. 1997; 10: 86-124. doi: 10.1128/ CMR.10.1.86

2. Denning DW, Invasive aspergillosis. Clin Infect Dis. 1998;26: 
781-803. doi: 10.1086/513943.

3. Latge JP. Aspergillosis fumigates and aspergillosis. Clin Microbiol Res. 1999;12:310-50. doi: 10.1128/CMR.12.2.310.

4. Ergin F, Arslan H, Azap A, Demirhan B, Karakayalı H, Haberal M. Invasive Aspergillosis in solid transplantation: report of eight cases and review of the literature. Traspl Int. 2003;16:280-6. doi: 10.1111/j.1432-2277.2003.tb00300.

5. Lin SJ, Schranz J, Teutsch SM. Aspergillosis case-fatality rate: systematic review of the literature. Clin Infect Dis. 2001;32: 358-66. doi: 10.1086/318483.

6. Soutani $\mathrm{AO}$, Chandrasakan $\mathrm{PH}$. The clinical spectrum of pulmonary aspergillosis. Chest. 2002;121:1988-99. doi: 10.1378/chest.121.6.1988.

7. Singh N, Avery RK, Munoz P, Pruett TL, Alexander B, Jacobs R, et al. Trends in risk profiles for and mortality associated with invasive aspergillosis among liver trans- plant recipients. Clin Infect Dis. 2003;36:46-52. doi: 10.1086/345441.

8. Weiland D, Ferguson RM, Peterson PK, Snover DC, Simmons RL, Najarian JS. Aspergillosis in 25 renal transplant patients. Epidemiology, clinical presentation, diagnosis, and management. Ann Surg. 1983;198:622-9. doi: 10.1097/00000658-198311000-00011
9. Linden E, Restrepo D, Dikman S, Murphy B, Huprikae S. Aspergillosis infection limited to renal allograft: case report and review of literature. Transpl Infect Dis. 2006;8:177-81. doi: 10.1111/j.1399-3062.2006.00134.x.

10. Tharayil John G, Shankar V, Talaulikar G, Mathews MS, Abraham Abraham M, Punnakuzhathil Thomas $P$, et al. Epidemiology of systemic mycoes among renal transplant recipients in India. Transplantation. 2003;75:1544-51. doi: 10.1097/01.tp.0000061610.34110.04.

11. Abbott KC, Hypolite I, Poropatich RK, Hshieh P, Cruess D, Hawkes CA. Hospitalizations for fungal infections after renal transplantation in the United States. Transpl Infect Dis. 2001: 3:203-11. doi: 10.1034/j.1399.

12. Bodey GP, Vartivarian S. Aspergillosis. Eur J Microbiol Infect Dis. 1989;8:413-37. doi: 10.1007/bf01964057.

13. Gottesdiener KM. Transplanted infections: donor-to-host transmission with the allograft. Ann Intern Med. 1989;110: 1001-16. doi: 10.7326/0003-4819-110-12-1001.

14. Estrada C, Desai AG, Chirch LM, Suh H, Seidman R, Darras $\mathrm{F}$, et al. Invasive aspergillosis in a renal transplant recipient successfully treated with interferon-gamma. Case Rep Transplant. 2012;2012:493758. doi: 10.1155/2012/493758. 